\title{
Optimizing Network Controllability with Minimum Cost
}

\author{
Xiao Wang $(\mathbb{D}$ and Linying Xiang $\mathbb{D}$ \\ School of Control Engineering, Northeastern University at Qinhuangdao, Qinhuangdao 066004, China \\ Correspondence should be addressed to Linying Xiang; xianglinying@neuq.edu.cn
}

Received 8 December 2020; Revised 6 January 2021; Accepted 13 January 2021; Published 27 January 2021

Academic Editor: Ning Cai

Copyright ( 2021 Xiao Wang and Linying Xiang. This is an open access article distributed under the Creative Commons Attribution License, which permits unrestricted use, distribution, and reproduction in any medium, provided the original work is properly cited.

\begin{abstract}
In this paper, the issue of optimally modifying the structure of a directed network to guarantee its structural controllability is investigated. Given a directed network, in order to obtain a structurally controllable system, a framework for finding the minimum number of directed edges that need to be added to the network is proposed. After we get these edge-addition configurations, we further calculate the network cost of each optimization scheme and choose the one with the minimum cost. Our main contribution is twofold: first, we provide an algorithm able to find all optimal network modifications in polynomial time; second, we provide a way to calculate the cost of optimizing the network based on the node betweenness. Numerical simulations are given to illustrate the theoretical results.
\end{abstract}

\section{Introduction}

The ultimate goal of complex network research is to find effective means to control network behavior and make it serve human beings. Controllability is a basic concept in control theory, which quantifies the ability to control a dynamical system from any initial state to any final state in finite time [1]. In the past decade, the issue of network controllability for complex dynamical systems has attracted increasing attention and becomes a focal topic in interdisciplinary research [2-30]. Numerous works have been reported from rather diverse perspectives on such topics as structural controllability [2, 3]; exact controllability [18]; edge dynamics [19-21]; optimization [22-24]; control energy [25, 26]; and robustness [27, 28].

In the study of network controllability, we usually rely on the theory of structural controllability [31-37]. If there is a matrix pair that is controllable, all structurally equivalent matrix pairs are controllable except for special ill-conditioned cases [31]. Recently, those results have been applied to the controllability analysis of directed complex networks $[2,3,16,19,22,23]$ from a graph-theoretic perspective. Note that it is very effective to analyze network controllability by using tools developed under the background of structural control theory [31].
Optimization of the network controllability is of prime importance in real applications. Generally speaking, given a network which is structurally uncontrollable, we can make it structurally controllable through two strategies: (i) add external input signals to the original network [16] and (ii) add new edges to the network topology [23]. Wang et al. provided a method to change the structure of a complex network to make the system structurally controllable when only a single driver node was considered [22]. Zhang and Zhou considered three related problems on determining the minimal cost structural perturbations, including edge additions, edge deletions, and input deletions to make a networked system structurally controllable/uncontrollable [24]. Chen et al. proposed an approach to adding minimum directed edges to the original network so as to ensure structural controllability [23].

Motivated by the above discussions, a minimum-cost optimization method to guarantee structural controllability is investigated in this paper. It should be emphasized that, differing from [23], in this work, a new method is proposed to optimize network topology and thus to ensure the network controllability. Moreover, it also provides a way to calculate the total cost of optimizing the network. However, in [23], it only gives a method to optimize the network 
topology without considering the optimization cost. Note that calculating the optimization cost is exactly the major point in this work. In [27], Zhang et al. considered the problem of network cost. Although the measurement index of edge cost was given therein, it did not provide a simple and effective method to calculate the total network cost. Compared with the previous works, we not only address the problem of optimizing network controllability but also propose a way to calculate the cost of optimizing the network. The main contributions of this article are as follows. (i) We propose a new method to optimize the network topology so as to ensure the network controllability. (ii) We propose an algorithm to solve the optimal edge-addition configuration problem. (iii) After getting all the edge-addition configurations, we introduce network cost measurement indexes to calculate the cost of optimizing the network. Based on which, we can determine the optimal edge-addition configuration with minimum-cost. The results of this paper can provide both theoretical and technical guidance for the analysis and control of real complex networks. The obtained results shed some lights on the transformation of a structurally uncontrollable network to a structurally controllable one with a low cost. For example, in the power network, transmission lines with the lowest cost can be set up among substations to safely and efficiently control the entire power network.

The rest of the paper is organized as follows. Section 2 introduces the notation and terminology used in this paper. Problem formulation and preliminaries on graph theory are introduced in Section 3. The main results are given in Section 4. In Section 5, a network cost index is given to determine the minimum-cost edge-addition configuration. Finally, the summary of this paper and the prospect of future research are presented in Section 6.

\section{Notations}

In this paper, $\mathbb{R}$ denotes the set of real numbers, $\mathbb{R}^{m}$ is the space of real $m$-vectors, and $\mathbb{R}^{m \times n}$ is the space of $m \times n$ real matrices. For a set $\mathcal{S}$, its cardinality is denoted by $|\mathcal{S}|$.

A directed graph $G=(V, E)$ consists of a node set $V=$ $\{1,2, \ldots, n\}$ and an edge set $E=\{(i, j)\}$. Here, $(i, j) \in E$ implies that there exists a directed edge from node $i$ to node $j$, and $i$ and $j$ are called the parent node and the child node, respectively. We can also say that the tail node $i$ is pointing toward the head node $j$. For a digraph $G$, a directed path of length $k+1$ from node $i$ to node $j$ is defined as a sequence of distinct edges of the form $\left(i, i_{1}\right),\left(i_{1}, i_{2}\right), \ldots,\left(i_{k}, j\right)$, in which all nodes $i, i_{1}, \ldots, i_{k}, j$ are distinct. Here, node $i$ is called the beginning node and $j$ the end node of the directed path. A node $i_{2} \in V$ is reachable from $i_{1} \in V$ if there exists a directed path in $G$ from $i_{1}$ to $i_{2}$. A directed graph $G_{s}=\left(V_{s}, E_{s}\right)$ is a subgraph of $G$ if $V_{s} \subseteq V$ and $E_{s} \subseteq E$. A directed graph is said to be strongly connected if there exists a directed path between any two nodes. A strongly connected component (SCC) is a maximal subgraph $G_{s}$ that is strongly connected. Particularly, a source SCC has no incoming edges from another SCC.
A digraph $G$ contains a dilation if there is a subset of nodes $S \subset V$ such that the common-neighbor set of $S$, denoted by $T(S)$, has fewer nodes than $S$ itself, i.e., $|T(S)|<|S|$. Here, $T(S)$ is the set of nodes $j$, in which there is a directed edge from node $j$ to some other node in $S$. Notice that a digraph $G$ contains no dilation if each node has its own independent parent node. It is intuitively plausible that a dilation is a subgraph containing a relatively large number of nodes that are "dominated" by a small number of other nodes.

\section{Problem Statement and Preliminaries}

Consider a linear time-invariant (LTI) networked dynamical system described by

$$
\dot{x}(t)=A x(t)+B u(t)
$$

where $x(t)=\left[x_{1}(t), x_{2}(t), \ldots, x_{n}(t)\right]^{T} \in \mathbb{R}^{n}$ is the state vector of all nodes; $u(t)=\left[u_{1}(t), u_{2}(t), \ldots, u_{m}(t)\right]^{T} \in \mathbb{R}^{m}$ is the input vector; $B=\left(b_{i j}\right) \in \mathbb{R}^{n \times m}$ is the input matrix identifying the nodes that are directly controlled, and $A=$ $\left(a_{i j}\right) \in \mathbb{R}^{n \times n}$ is the adjacency matrix of the underlying network. The overall networked system described by (1) can be denoted by the matrix pair $(A, B)$.

Definition 1. Linear network (1) is said to be state controllable if, for any initial state $x\left(t_{0}\right) \in \mathbb{R}^{n}$ and any final state $x\left(t_{f}\right) \in \mathbb{R}^{n}$, there exist a finite time $t_{1}$ and an input $u(t) \in \mathbb{R}^{m}, t \in\left[t_{0}, t_{1}\right]$, such that $x\left(t_{1} ; x\left(t_{0}\right), u\right)=x\left(t_{f}\right)$.

If networked system (1) is state controllable, we can say that the matrix pair $(A, B)$ is state controllable.

Definition 2 (see $[16,31]$ ). A linear control system $(A, B)$ is a structured system if the elements in $A$ and $B$ are either fixed zeros or independent nonzero parameters. Both the two matrices $A$ and $B$ are called structured matrices.

In this paper, it is assumed that we only know the structure of the matrices $A$ and $B$. This means that we know which elements in the matrices are fixed to zero and consequently which elements are nonzero free parameters.

Definition 3. A linear control system $(A, B)$ is structurally controllable if we can set some values to the nonzero parameters in $A$ and $B$ such that the resulting system is state controllable in the sense of Kalman defined in Definition 1.

A structured system can be represented by a directed graph whose nodes denote the (state and input) variables and edges indicate the connections between some variables [31]. In this paper, a structured system $(A, B)$ is denoted by a directed graph $G(A, B)=(V, E)$, in which $V=V_{A} \cup V_{B}$ is the node set and $E=E_{V_{A}, V_{A}} \cup E_{V_{B}, V_{A}}$ is the edge set. In particular, $V_{A}=\left\{x_{1}, x_{2}, \ldots, x_{n}\right\}$ is the set of state nodes, corresponding to the $n$ nodes in the original network; $V_{B}=$ $\left\{u_{1}, u_{2}, \ldots, u_{m}\right\}$ is the set of input nodes corresponding to the $m$ inputs; $E_{V_{A}, V_{A}}=\left\{\left(x_{i}, x_{j}\right) \mid a_{j i} \neq 0\right\}$ is the set of edges 
between state nodes; and $E_{V_{B}, V_{A}}=\left\{\left(u_{i}, x_{j}\right) \mid b_{j i} \neq 0\right\}$ is the set of edges between input nodes and state nodes. In the whole paper, suppose that any input signal is applied to only one node, referred to as a driver node. A state node being reachable means that there is a directed path from some input node to this state node. Similarly, a node set is reachable if each node in the set is reachable. Notice that, in the remaining of the paper, unless otherwise specified, the reachability is only used for the state nodes.

In a digraph, an edge subset $\widetilde{M}$ is a matching if no two edges in $\tilde{M}$ share a common parent node or a common child node. A matching of maximum size is called a maximum matching. The maximum matching of a digraph can be denoted by mapping the digraph to its bipartite representation. Consider a directed network $G(A, B)$, whose bipartite representation can be described by $\mathscr{B}(A, B)=\mathscr{B}\left(V_{A}^{+} \cup\right.$ $\left.V_{B}, V_{A}^{-}, E_{V_{A}^{+}, V_{A}^{-}} \cup E_{V_{B}, V_{A}^{-}}\right)$, in which $V_{A}^{+}=\left\{x_{1}^{+}, x_{2}^{+}, \ldots, x_{n}^{+}\right\}$and $V_{A}^{-}=\left\{x_{1}^{-}, x_{2}^{-}, \ldots, x_{n}^{-}\right\}$. That is, each state node $x_{i}$ of the original digraph is split into two nodes $x_{i}^{+}$and $x_{i}^{-}$. Here, $\left\{x_{i}^{+}, x_{j}^{-}\right\} \in E_{V_{A}^{+}, V_{A}^{-}}$if $\left(x_{i}, x_{j}\right) \in E_{V_{A}, V_{A}}$ and $\left\{u_{i}, x_{j}^{-}\right\} \in E_{V_{B}, V_{A}^{-}}$if $\left(u_{i}, x_{j}\right) \in E_{V_{B}, V_{A}}$. To describe the relationship between the digraph and its bipartite graph, we use a signal-notation mapping $f: E_{V_{A}, V_{A}} \cup E_{V_{B}, V_{A}} \longrightarrow E_{V_{A}^{+}, V_{A}^{-}} \cup E_{V_{B}, V_{A}^{-}}$to map directed edges from the system digraph into undirected edges of the system bipartite graph as follows: $f\left(\left(u_{i}, x_{j}\right)\right)=$ $\left\{u_{i}, x_{j}^{-}\right\}$and $f\left(\left(x_{i}, x_{j}\right)\right)=\left\{x_{i}^{+}, x_{j}^{-}\right\}$. Also, we have that $f^{-1}\left(\left\{u_{i}, x_{j}^{-}\right\}\right)=\left(u_{i}, x_{j}\right)$ and $f^{-1}\left(\left\{x_{i}^{+}, x_{j}^{-}\right\}\right)=\left(x_{i}, x_{j}\right)$.

Definition 4. The element $r_{i j}=1$ in the matrix $R \in \mathbb{R}^{n \times n}$ if there is a directed path from node $i$ to node $j(i \neq j)$. Set $r_{i i}=1, i=1,2, \ldots, n$. The matrix $R$ is called reachable matrix.

If only one external input is applied to node 1 , then the first row of the matrix $R$ can be used to determine which nodes are unreachable.

Definition 5. The element $p_{i j}=1$ in the matrix $P$ if edge $(i, j)$ is one of the matching edges of a maximum matching about a bipartite graph. The matrix $P$ is called maximum matching matrix.

The maximum matching of a directed graph is not unique. Therefore, the corresponding maximum matching matrix $P$ is not unique. It can be found from the matrix $P$ that the number of nonzero elements in the matrix $P$ is the number of matching edges in the maximum matching, and each row and each column have at most one nonzero element. The $j^{\text {th }}$ column is full of zero elements, indicating that node $j$ in the network does not have its own independent parent node.

Definition 6. Consider a directed network, in which only one external input signal is applied to node 1 . If $n=\sum_{j=1}^{n} r_{1 j}$, $r_{1 j} \in R$, then such reachable matrix $R$ is called $1-R$ matrix. For example,

$$
1-R=\left[\begin{array}{lllll}
1 & 1 & 1 & 1 & 1 \\
0 & 1 & 0 & 0 & 0 \\
1 & 1 & 1 & 1 & 0 \\
0 & 0 & 0 & 1 & 0 \\
0 & 0 & 0 & 1 & 1
\end{array}\right] .
$$

Obviously, if the reachable matrix $R$ of a network is a $1-R$ matrix, then all the state nodes in the network are reachable.

Definition 7. Consider a directed network, in which only one external input signal is applied to node 1 . If the maximum matching matrix $P$ has a unique nonzero element in each column except for the first column, then such maximum matching matrix $P$ is called $1-P$ matrix. For example,

$$
1-P=\left[\begin{array}{lllll}
0 & 1 & 0 & 0 & 0 \\
0 & 0 & 1 & 0 & 0 \\
0 & 0 & 0 & 1 & 0 \\
0 & 0 & 0 & 0 & 1 \\
0 & 0 & 0 & 0 & 0
\end{array}\right] \text {. }
$$

Obviously, if the maximum matching matrix $P$ of a network is $1-P$ matrix, then there is no dilation in the network.

A necessary and sufficient condition for the structural controllability of an LTI system is given as follows [31].

Lemma 1 (see [31]). The pair $(A, B)$ is structurally controllable if and only if the following two conditions are satisfied simultaneously:

(1) Every state node $x \in V_{A}$ in the digraph $G(A, B)=$ $\left(V_{A} \cup V_{B}, E_{V_{A}, V_{A}} \cup E_{V_{B}, V_{A}}\right)$ is reachable from some input node $u \in V_{B}$

(2) The digraph $G(A, B)$ contains no dilations

Then, we have the following controllability criterion.

Theorem 1. A directed network $G(A, B)$ with $B=\left[b_{1}, 0, \ldots, 0\right]^{T}$ is structurally controllable if and only if the following two conditions are satisfied simultaneously:

(1) The reachable matrix of $G(A, B)$ is a $1-R$ matrix

(2) The maximum matching matrix of $G(A, B)$ is a $1-P$ matrix

In this paper, given a structurally uncontrollable directed network, we study the problem of adding the least edges to improve the topology so as to obtain a structurally controllable system. After we get these optimal edge-addition configurations, we need to calculate the network cost of each optimization scheme and choose the one with the minimum cost. In summary, the problem is given as follows. 
Problem 1. Given the pair $(A, B)$ with $B=\left[b_{1}, 0, \ldots, 0\right]^{T}$, find

$$
\widetilde{A}^{*}=\arg \min _{\widetilde{A} \in\{0,1\}^{n \times n}}\|\widetilde{A}\|_{0},
$$

s.t. the reachable matrix of digraph $G(A+\widetilde{A}, B)$ is a $1-R$ matrix and the maximum matching matrix is a $1-P$ matrix,

where $\|\widetilde{A}\|_{0}$ denotes the number of nonzero elements in a matrix $\widetilde{A}$.

If $(A+\widetilde{A}, B)$ is structurally controllable, we refer to the matrix $\widetilde{A}$ as an effective perturbed matrix and to $\widetilde{A}^{*}$ in (4) as the modified matrix. The aim of this paper is to provide a characterization of all possible modified matrices by using graph-theoretical tools and design an algorithm to obtain such a solution.

\section{Network Topology Optimization to Ensure Structural Controllability}

Note that the system digraph is denoted by $G(A, B)=$ $\left(V_{A} \cup V_{B}, E_{V_{A}, V_{A}} \cup E_{V_{B}, V_{A}}\right)$. Therefore, given an effective perturbed matrix $\widetilde{A}$, we can relate a digraph to the perturbed structured system $(A+\widetilde{A}, B)$, which we denote by $G(A+$ $\widetilde{A}, B)=\left(V_{A} \cup V_{B}, E_{V_{A}, V_{A}} \cup E_{V_{B}, V_{A}} \cup \underset{\widetilde{E}}{\widetilde{E}}\right)$, where the edge set $\widetilde{E} \subseteq V_{A} \times V_{A}$ is such that $\left(x_{i}, x_{j}\right) \in \widetilde{E}$ if and only if $\widetilde{a}_{j i}=1$. Since the matrix $\widetilde{A}$ is closely related to the $\widetilde{E}$, we can rewrite Problem 1 in a different way.

Problem 2. Given the system digraph $G(A, B)=\left(V_{A} \cup V_{B}\right.$, $\left.E_{V_{A}, V_{A}} \cup E_{V_{B}, V_{A}}\right)$ with $B=\left[b_{1}, 0, \ldots, 0\right]^{T}$, find

$$
\widetilde{E}^{*}=\arg \min _{\tilde{E} \subseteq V_{A} \times V_{A}}|\widetilde{E}|,
$$

s.t. the reachable matrix of the digraph $G(A+\widetilde{A}, B)=$ $\left(V_{A} \cup V_{B}, E_{V_{A}, V_{A}} \cup E_{V_{B}, V_{A}} \cup \widetilde{E}\right)$ is a $1-R$ matrix and the maximum matching matrix is a $1-P$ matrix.

Additionally, define a feasible edge-addition configuration as a set of directed edges that is a feasible solution of Problem 2.

The solutions to Problem 2 are given in this section. First, a definition is introduced to describe the smallest set of edges needed to achieve reachability, i.e., satisfy condition (1) in Lemma 1. Let $G(A, B)=\left(V_{A} \cup V_{B}, E_{V_{A}, V_{A}} \cup E_{V_{B}, V_{A}}\right)$ be the system digraph. The set of state nodes $V_{A}$ can be divided into two sets based on their reachability, namely, $V_{A}=\bar{R} \cup \bar{N}$, where $\bar{R}$ is the set of reachable nodes and $\bar{N}$ is the set of unreachable nodes. In addition, assume that there are $r$ source SCCs that are unreachable, whose node sets are denoted by $N_{1}, N_{2}, \ldots, N_{r} \subseteq \bar{N}$. In order to make the nodes in these unreachable source SCCs reachable, we need to add a new edge between the reachable node and the node in the source SCC so that all the nodes in the source SCC are reachable. Moreover, since the source SCC has outgoing edges pointing to other nodes, the unreachable nodes that are connected to the source SCC will also become reachable.
Definition 8. A set $S_{E}$ is made up of connected edges, then the set $S_{E}$ is called the connected edge set. Here, the connected edge refers to the connecting edge between the reachable node and the unreachable node.

Algorithm 1 is illustrated in Figure 1. The connected edge set contains the minimum number of added edges required to ensure that all the state nodes are reachable. Obviously, the connected edge set can only satisfy condition (1) in Lemma 1 and cannot guarantee the structural controllability of the networked system. To ensure structural controllability of the system, these edge additions must satisfy two conditions: (i) a set of connected edges and (ii) the "tail" node of the new edge is not used as an independent parent node in the maximum matching. It is the "head" node of the edge that has no independent parent node.

Theorem 2. Consider a directed network $G(A, B)$, whose bipartite representation is denoted by $\mathscr{B}(A, B)$. Let $M$ be a maximum matching, $U_{o}(M)=\left\{v_{i}^{o}: i \in\left\{1,2, \ldots, n_{o}\right\}\right\}$ be a node set in which each node is not used as independent parent node, and $U_{r}(M)=\left\{v_{i}^{r}: i \in\left\{1,2, \ldots, n_{r}\right\}\right\}$ be a node set with no independent parent nodes. $A$ set $\widetilde{E}$ is a feasible edge-addition configuration if and only if it contains the union of the following two sets:

(1) $S_{E}$ is the set of connected edges

(2) $S_{M}=\left\{f^{-1}\left(\left\{v_{i}^{o}, v_{i}^{r}\right\}\right): v_{i}^{o} \in U_{o}(M), v_{i}^{r} \in U_{r}(M)\right.$, $\left.i=\left\{1,2, \ldots, n_{r}\right\}\right\}$

Theorem 2 provides some feasible edge-addition configurations, but we need to find the optimal one from these configurations. Therefore, the first task is to select the optimal solution from these feasible solutions. From the above discussion, it can be found that, after determining the maximum matching of a bipartite graph, if those unmatched nodes (nodes without independent parent nodes) happen to be distributed in different source SCCs, then the added edges just meet both conditions in Lemma 1, which is exactly what is needed. To explore this situation, we introduce the following concepts.

Definition 9. Consider a directed network $G(A, B)$, whose bipartite representation is denoted by $\mathscr{B}(A, B)$. Let $M$ be a maximum matching associated with $\mathscr{B}(A, B)$. Moreover, let $U_{r}(M)$ be the set of nodes in which each node has no independent parent nodes. If there is at least one node $i$, $i \in U_{r}(M)$ in an unreachable source SCC, then such an unreachable source SCC is called an ideal source SCC.

Whether an unreachable source SCC is an ideal source SCC depends mainly on the specific maximum matching. Because there may be more than one maximum matching corresponding to a directed network, it is not possible to determine whether a node has an independent parent node in the maximum matching.

Definition 10. The $N_{s}$ of the directed network $G(A, B)$ is defined as the maximum number of ideal source SCCs in all the maximum matchings. 
Input: reachable nodes sets $\bar{R}$ and unreachable nodes sets $\bar{N}$

(1) Order the unreachable source SCCs: $N_{1}, N_{2}, \ldots, N_{r}$

(2) Select any edge $(i, j)$ in which $i$ is in the set of reachable nodes and $j$ is in the first source SCC

(3) Merge all reachable state nodes into a larger set (we can do it using either BFS/DFS or union-find)

(4) Call Steps 2-3 recursively until all unreachable source SCCs become reachable

Algorithm 1: Set of connected edges.

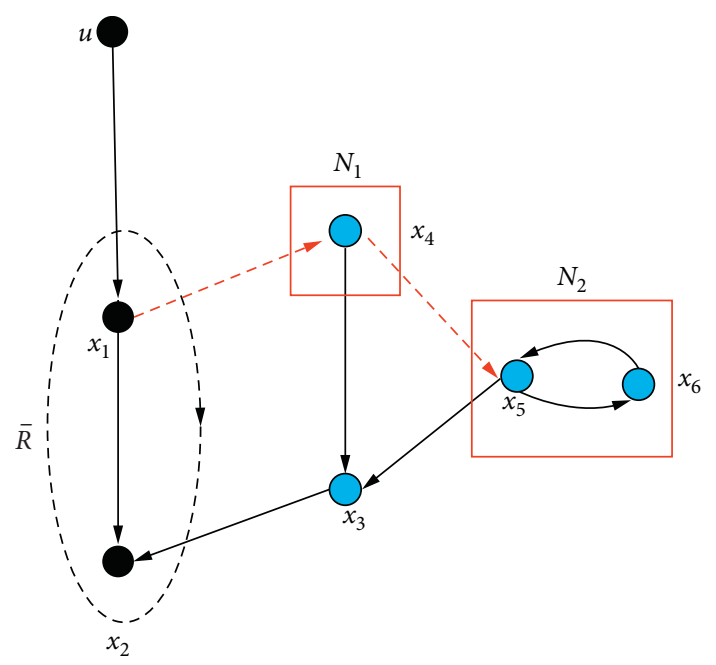

(a)

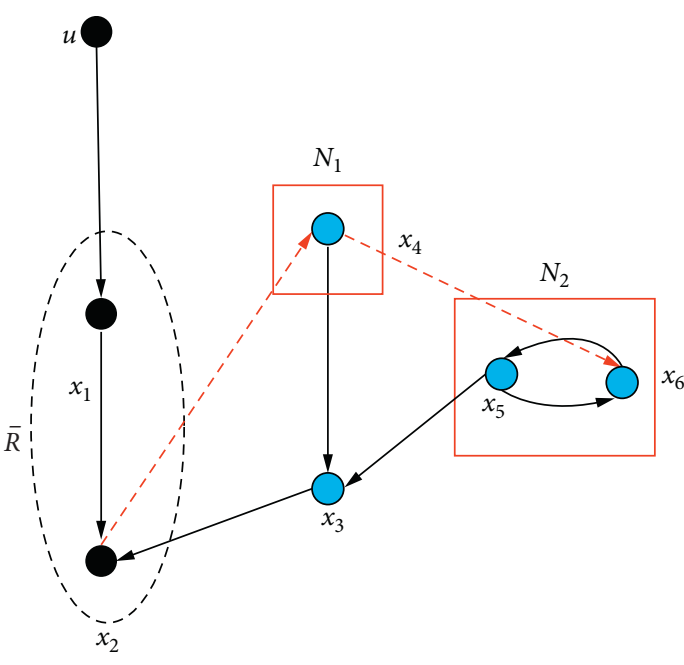

(b)

Figure 1: Illustration of Algorithm 1. The black and blue nodes and all the black edges consist of the original directed network $G(A, B)$. The black nodes, except for the input node $u$, constitute the set of reachable state nodes $\bar{R}=\left\{x_{1}, x_{2}\right\}$. Blue nodes constitute the set of unreachable state nodes $\bar{N}=\left\{x_{3}, x_{4}, x_{5}, x_{6}\right\}$. There are two unreachable state source SCCs, $N_{1}$ and $N_{2}$. In (a), we give a possible edge-addition configuration for Algorithm 1. First, we add edge $\left(x_{1}, x_{4}\right)$ to $S_{E}$. Then, the state node $x_{4}$ from $N_{1}$ becomes reachable, and thus the state node $x_{3}$ becomes reachable. Next, we add edge $\left(x_{4}, x_{5}\right)$ to $S_{E}$, and then the state nodes $x_{5}$ and $x_{6}$ from $N_{2}$ become reachable, i.e., $S_{E}=\left\{\left(x_{1}, x_{4}\right),\left(x_{4}, x_{5}\right)\right\}$. In (b), we add edges $\left(x_{2}, x_{4}\right)$ and $\left(x_{4}, x_{6}\right)$ to $S_{E}^{\prime}$, i.e., $S_{E}^{\prime}=\left\{\left(x_{2}, x_{4}\right),\left(x_{4}, x_{6}\right)\right\}$. Therefore, $S_{E}$ and $S_{E}^{\prime}$ are two possible sets of connected edges.

We can determine a maximum matching attaining $N_{s}$ using Algorithm 2.

We take Figure 2, for example, to illustrate Algorithm 2.

The reachable matrix corresponding to the digraph in Figure 2(a) is expressed as follows:

$$
R=\left[\begin{array}{llllll}
1 & 1 & 0 & 1 & 0 & 0 \\
0 & 1 & 0 & 0 & 0 & 0 \\
1 & 1 & 1 & 1 & 0 & 0 \\
0 & 0 & 0 & 1 & 0 & 0 \\
0 & 0 & 0 & 1 & 1 & 1 \\
0 & 0 & 0 & 1 & 1 & 1
\end{array}\right] .
$$

The unreachable node set can be determined as $\bar{N}=$ $\left\{x_{3}, x_{5}, x_{6}\right\}$ by the position of the 0 element in the first row of $R$. Moreover, there are two unreachable source SCCs (red box), whose node sets are $N_{1}=\left\{x_{3}\right\}$ and $N_{2}=\left\{x_{5}, x_{6}\right\}$, respectively. Then, we can label columns 3, 5, and 6 of $R$ as follows:

$$
R=\left[\begin{array}{llllll} 
& & * & & * & * \\
1 & 1 & 0 & 1 & 0 & 0 \\
0 & 1 & 0 & 0 & 0 & 0 \\
1 & 1 & 1 & 1 & 0 & 0 \\
0 & 0 & 0 & 1 & 0 & 0 \\
0 & 0 & 0 & 1 & 1 & 1 \\
0 & 0 & 0 & 1 & 1 & 1
\end{array}\right] .
$$

Figure 2(b) shows the bipartite representation of the original directed network (Figure 2(a)). In order to make the column ordinals corresponding to all 0 columns in the maximum matching matrix $P$ coincide with the marked column ordinals as much as possible, an ideal maximum matching $M$ is determined in Figure 2(c), and its corresponding maximum matching matrix is expressed as follows: 
Input: A directed network $G(A, B)$ with $B=\left[b_{1}, 0, \ldots, 0\right]^{T}$;

(1) Write the reachable matrix $R$ of the directed network, and determine the unreachable node set $\bar{N}$ in the network by the position (column ordinal) of the 0 element in the first row.

(2) Find the unreachable source SCCs.

(3) Select the nodes located in the source SCCs from the unreachable nodes set $\bar{N}$ and mark their column ordinals.

(4) By using the marked column ordinals to identify an ideal maximum matching $M$. Its corresponding maximum matching matrix is $P^{*}$. The column ordinals corresponding to all 0 columns in the matrix $P^{*}$ need to match the marked column ordinals as much as possible.

(5) According to Step 3, an ideal maximum matching matrix $P^{*}$ can be obtained. From the matrix $P^{*}$, the nodes corresponding to the matching column ordinals can be found.

(6) Based on the distribution of the nodes found in Step 5 in the source SCCs, $N_{s}$ can be calculated.

Algorithm 2: Determine the ideal maximum matching to get $N_{s}$.

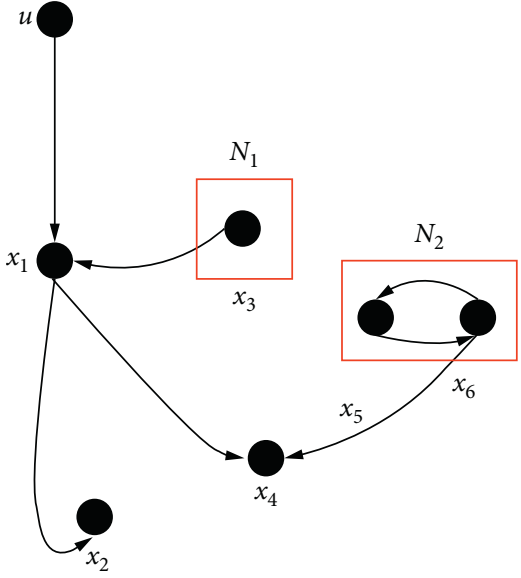

(a)

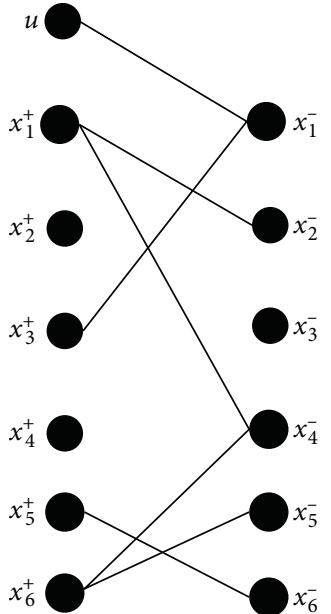

(b)

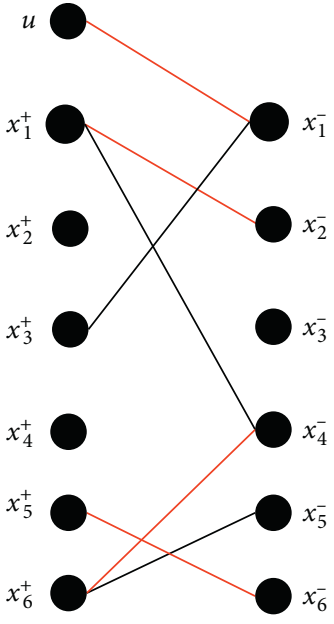

(c)

Figure 2: Example illustrating Algorithm 2.

$$
P^{*}=\left[\begin{array}{lllllll}
1 & 1 & 0 & 1 & 0 & 0 \\
0 & 1 & 0 & 0 & 0 & 0 \\
1 & 1 & 1 & 1 & 0 & 0 \\
0 & 0 & 0 & 1 & 0 & 0 \\
0 & 0 & 0 & 1 & 1 & 1 \\
0 & 0 & 0 & 1 & 1 & 1
\end{array}\right] .
$$

There are at most two 0 columns in $P^{*}$ that are consistent with the marked column ordinals, and the corresponding node $x_{3}$ is located in $N_{1}$, and node $x_{5}$ is located in $N_{2}$, so $N_{s}=2$.

If all the state nodes that are not used as independent parent nodes are unreachable, then additional edges are needed to satisfy condition (1) in Lemma 1 . Therefore, in this case, calculating $N_{s}$ according to Algorithm 2 does not necessarily lead to an optimal configuration of added edges. To illustrate this statement, we take Figure 3 for example.

Next, we will propose Algorithm 3 to solve Problem 2. Algorithm 3 is mainly divided into the following four steps:
Step 1. All the state nodes in the directed network are classified into a reachable node set and an unreachable node set, respectively, based on the node reachability.

Step 2. Determine the ideal maximum matching to get $N_{s}$. If there exist some unreachable nodes that are not used as independent parent nodes in the ideal maximum matching, then we alter the matching by finding a directed path rooted at the input node.

Step 3. Add some edges to satisfy Lemma 1. These edges start at reachable nodes that are not used as independent parent nodes and end at nodes that have no independent parent nodes in unreachable source SCCs.

Step 4. If there are unreachable nodes that are not used as independent parent nodes, then we need to add a set of connected edges to ensure that both two conditions of Lemma 1 are satisfied.

Given a structurally uncontrollable system $(A, B)$ that contains unreachable nodes and/or dilations. Therefore, we need to optimize the network topology to ensure structural controllability by adding edges. Algorithm 3 is given to obtain optimal edge-addition configuration to solve Problem 2. 


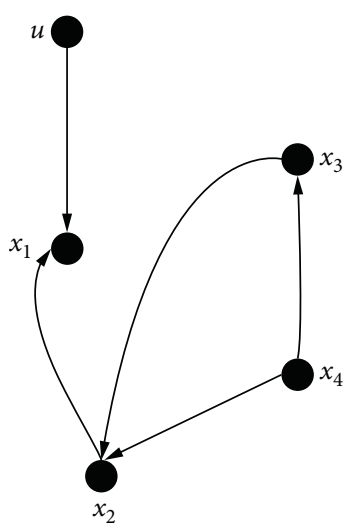

(a)

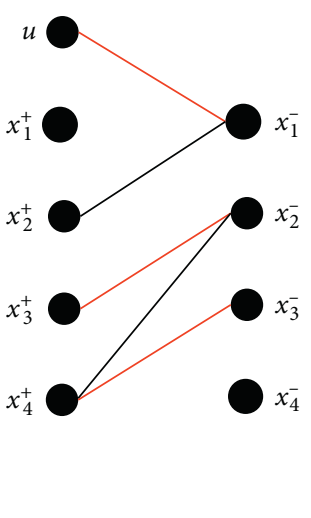

(b)

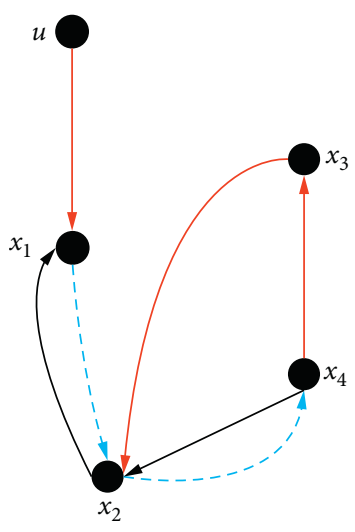

(c)

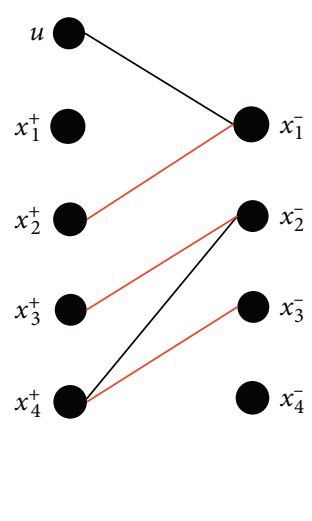

(d)

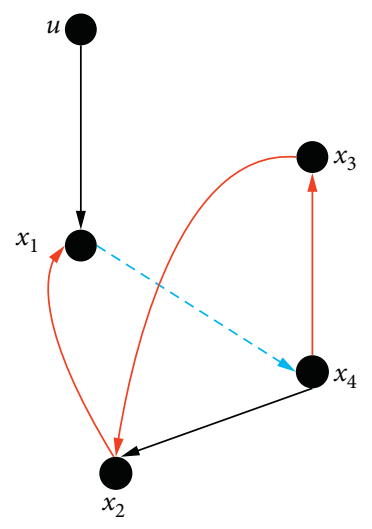

(e)

FIgURE 3: The maximum matching of a directed graph is not unique, and different maximum matchings will result in different feasible edgeaddition configurations. In (a), the initial system digraph $G(A, B)$ is given. The red edges in (b) and (d) form two different maximum matchings. The red edges in (c) and (e) are determined by the maximum matchings in (b) and (d), respectively. In (c), after determining the maximum matching, node $x_{4}$ has no independent parent node and node $x_{2}$ has not been used as the parent node. So, we need to add the edge $\left(x_{2}, x_{4}\right)$ to satisfy condition (2) of Lemma 1 . Since node $x_{2}$ is unreachable, we also need to add the edge $\left(x_{1}, x_{2}\right)$ to satisfy condition (1) of Lemma 1 . Then, we have $\widetilde{E}_{1}=\left\{\left(x_{1}, x_{2}\right),\left(x_{2}, x_{4}\right)\right\}$. In (e), after determining the maximum matching, node $x_{4}$ has no independent parent node and node $x_{1}$ has not been used as the parent node. So, we can add edge $\left(x_{1}, x_{4}\right)$ to satisfy both two conditions of Lemma 1 , i.e., $\widetilde{E}_{2}=\left\{\left(x_{1}, x_{4}\right)\right\}$. Therefore, $\widetilde{E}_{2}$ is an optimal edge-addition configuration but $\widetilde{E}_{1}$ is not.

Input: A directed network $G(A, B)$;

(1) All the state nodes in the network are classified into a reachable node set $\bar{R}$ and an unreachable node set $\bar{N}$. Then, determine the unreachable source SCCs in the directed network $G(A, B)$.

(2) Using Algorithm 2 to get $M^{\prime}$ and $N_{s}$.

(3) if $U_{o}\left(M^{\prime}\right) \cap \bar{R}=\varnothing$, then

(4) Find an unreachable node $x_{j}$, and thus add the edge $\left(x_{i}, x_{j}\right), x_{i} \in \bar{R}$;

(5) $M \leftarrow M^{\prime} \cup\left\{\left(x_{i}, x_{j}\right)\right\}$;

(6) else

(7) Set $M=M^{\prime}$;

(8) end if

(9) Obtain the unique set of disjoint directed paths $L=\cup_{i=1}^{q} L_{i}$ in $M$, where the beginning node of each $L_{i}$ is in some unreachable source SCCs and the end node is not used as a separate parent node;

(10) Let $Q=\left\{q_{1}, q_{2}, \ldots, q_{n}\right\}$ and $Z=\left\{z_{1}, z_{2}, \ldots, z_{n}\right\}, q_{i}, z_{i}$ are the beginning and end nodes of each path $L_{i}$, respectively;

(11) Let $\widetilde{E}^{*} \leftarrow \varnothing, k \leftarrow 1$;

(12) if $Z \cap \bar{R}=\varnothing$, then

(13) Find a reachable node $v_{o}, v_{o} \in U_{o}(M)$;

(14) for $k \leq q$ do

(15) $\widetilde{E}^{*} \leftarrow \widetilde{E} \cup\left\{v_{k-1}, q_{k}\right\} ; k \leftarrow k+1$;

(16) $Z \subseteq \bar{R}$

(17) end for

(18) if $x_{i}^{+} \in U_{o}(M), U_{r}(M) \neq \varnothing$, then

(19) $\widetilde{E}^{*} \leftarrow \widetilde{E} \cup\left\{\left(x_{i}, x_{j}\right)\right\}, x_{j}^{-} \in U_{r}(M)$;

(20) $U_{o}(M) \leftarrow U_{o}(M) \backslash x_{i}^{+} ; U_{r}(M) \longleftarrow U_{r}(M) \backslash x_{j}^{-}$;

(21) when $U_{r}(M)=\varnothing$

(22) end if

Algorithm 3: Minimal edge addition.

Next, an example in Figure 4 is given to illustrate Algorithm 3.

\section{Network Optimization Cost}

We have solved the optimal edge-addition configuration problem; however, there are multiple potential edge- addition configurations to ensure structural controllability. From the application perspective, the lowest cost configuration is usually selected as the final optimization solution. Therefore, we present Problem 3 based on Problem 2, taking the network cost into account. In order to solve Problem 3, we introduce an edge cost measurement index to calculate the edge cost and thus obtain the cost of the whole network. 

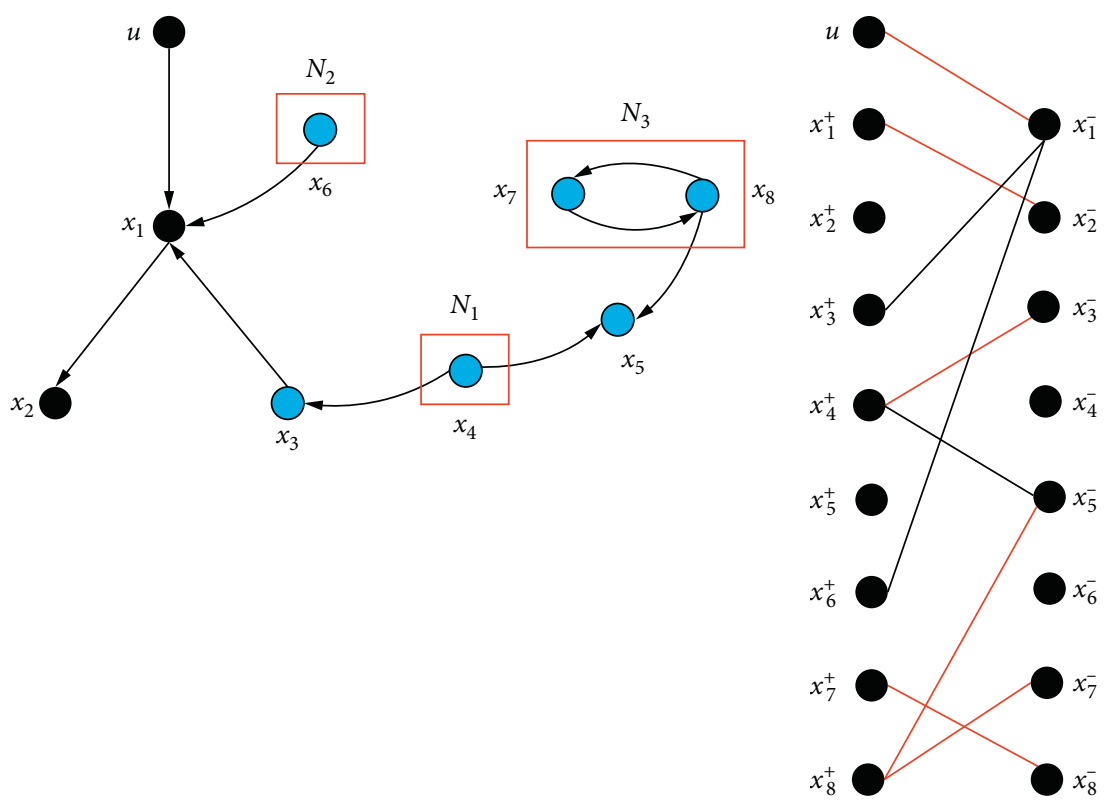

(a)

(b)

Figure 4: In (a), the directed network $G(A, B)$ contains a single input node $u$ and eight state nodes $x_{1}, x_{2}, \ldots, x_{8}$. We will first decompose the directed graph according to the first step of Algorithm $3, \bar{R}=\left\{x_{1}, x_{2}\right\}, \bar{N}=\left\{x_{3}, x_{4}, x_{5}, x_{6}, x_{7}, x_{8}\right\}$. There are three unreachable source SCCs in the digraph, $N_{1}=\left\{x_{4}\right\}, N_{2}=\left\{x_{6}\right\}, N_{3}=\left\{x_{7}, x_{8}\right\}$. In (b), we provide $\mathscr{B}(A, B)$ the bipartite graph of the directed graph to attain a maximum matching $M^{\prime}$ (red edges) according to Step 2 of Algorithm 3, i.e., $M^{\prime}=\left\{\left(u, x_{1}\right),\left(x_{1}, x_{2}\right),\left(x_{4}, x_{3}\right),\left(x_{8}, x_{5}\right),\left(x_{7}, x_{8}\right)\right\}$. According to the maximum matching, nodes $x_{4}, x_{6}$, and $x_{7}$ have no independent parent node, $U_{r}\left(M^{\prime}\right)=\left\{x_{4}, x_{6}, x_{7}\right\}$. The nodes $x_{2}, x_{3}, x_{5}$, and $x_{6}$ are not used as the independent parent node, $U_{o}\left(M^{\prime}\right)=\left\{x_{2}, x_{3}, x_{5}, x_{6}\right\}$. According to Step 3 of Algorithm 3, reachable node $x_{2}$ is not used as the parent node, $x_{2} \in \bar{R}, x_{3}, x_{5}, x_{6} \in \bar{N}$. Therefore, we need to pick nodes in $U_{o}\left(M^{\prime}\right)$ and $U_{r}\left(M^{\prime}\right)$, respectively, and they form edges that make nodes $x_{3}, x_{5}$, and $x_{6}$ become reachable. The added edge $\left(x_{2}, x_{4}\right)$ can satisfy the above conditions $\left(U_{o}(M) \subseteq \bar{R}\right)$. After the edge $\left(x_{2}, x_{4}\right)$ is added, a new maximum matching $M=M^{\prime} \cup\left\{\left(x_{2}, x_{4}\right)\right\}$ is formed. Then, the remaining set in which each node has not been used as an independent parent node is $U_{o}(M)=\left\{x_{5}, x_{3}, x_{6}\right\}$. The set with no independent parent node is $U_{r}(M)=\left\{x_{6}, x_{7}\right\}$. According to Step 18 of Algorithm 3, $U_{r}(M)=\left\{x_{6}, x_{7}\right\} \neq \varnothing$. So, we need to keep adding edges until the two conditions of Lemma 1 are satisfied. The other two edges added have four choices: $\left\{\left(x_{5}, x_{6}\right),\left(x_{3}, x_{7}\right)\right\},\left\{\left(x_{5}, x_{7}\right),\left(x_{3}, x_{6}\right)\right\},\left\{\left(x_{5}, x_{6}\right),\left(x_{6}, x_{7}\right)\right\}$, and $\left\{\left(x_{3}, x_{6}\right),\left(x_{6}, x_{7}\right)\right\}$. Finally, after adding three edges to the graph, both two conditions of Lemma 1 are satisfied, and thus a new directed graph $G(A+\widetilde{A}, B)$ is obtained. In summary, we can get four optimal edge-addition configurations as follows: $\widetilde{E}_{1}^{*}=\left\{\left(x_{2}, x_{4}\right),\left(x_{5}, x_{6}\right),\left(x_{3}, x_{7}\right)\right\}, \widetilde{E}_{2}^{*}=\left\{\left(x_{2}, x_{4}\right),\left(x_{5}, x_{7}\right),\left(x_{3}, x_{6}\right)\right\}$, $\widetilde{E}_{3}^{*}=\left\{\left(x_{2}, x_{4}\right),\left(x_{5}, x_{6}\right),\left(x_{6}, x_{7}\right)\right\}$, and $\widetilde{E}_{4}^{*}=\left\{\left(x_{2}, x_{4}\right),\left(x_{6}, x_{7}\right),\left(x_{3}, x_{6}\right)\right\}$ (a solution to Problem 2).

In addition, we need to adopt a simple and practical method to calculate the cost of the network and determine a minimum-cost configuration to ensure the controllability based on the optimal edge-addition configuration.

Problem 3. Consider a directed network $G(A, B)$, find

$$
\widetilde{E}^{*}=\arg \min _{\tilde{E} \subseteq V_{A} \times V_{A}}|\widetilde{E}|,
$$

s.t. the new directed network $G(A+\widetilde{A}, B)$ contains neither unreachable nodes nor dilations. Also, the cost of the new directed network must be the lowest one.

5.1. Main Idea. Given a structurally uncontrollable directed network $G(A, B)$. The optimal edge-addition configuration is obtained by using Algorithm 3. The first step of calculating the network optimization cost is to obtain the load of each node in the network. Note that the nature of node load is exactly consistent with the betweenness centrality of the node. Betweenness centrality of a node refers to the proportion of the number of paths passing through the node in the total number of shortest paths. Intuitively, the betweenness centrality reflects the importance of the node as a "bridge." Therefore, the initial load on each node can be denoted by its betweenness centrality [27]. We can calculate the betweenness centrality of each node by "pajek" software after importing a directed network. There is a nonlinear relationship between the load of a node and its capacity [38, 39], so we can determine the node capacity by this nonlinear relation. The cost of a node can be measured by its node capacity in the network. We take the larger one of the two node capacities as the cost of the edge that connects these two nodes [40]. In this paper, we calculate the network costs of all optimal edge-addition configurations and then choose the one with the lowest network cost as the optimal edge-addition configuration.

The specific calculation process of network cost is given as follows:

Step 1. Node load can be measured by the betweenness centrality 


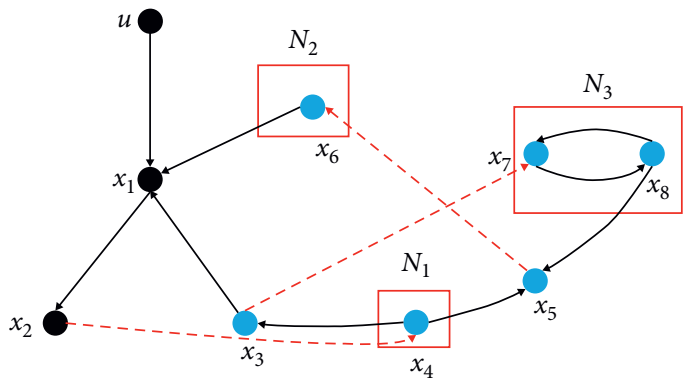

Figure 5: The new directed network resulting from the first configuration scheme $\widetilde{E}_{1}^{*}=\left\{\left(x_{2}, x_{4}\right),\left(x_{5}, x_{6}\right),\left(x_{3}, x_{7}\right)\right\}$.

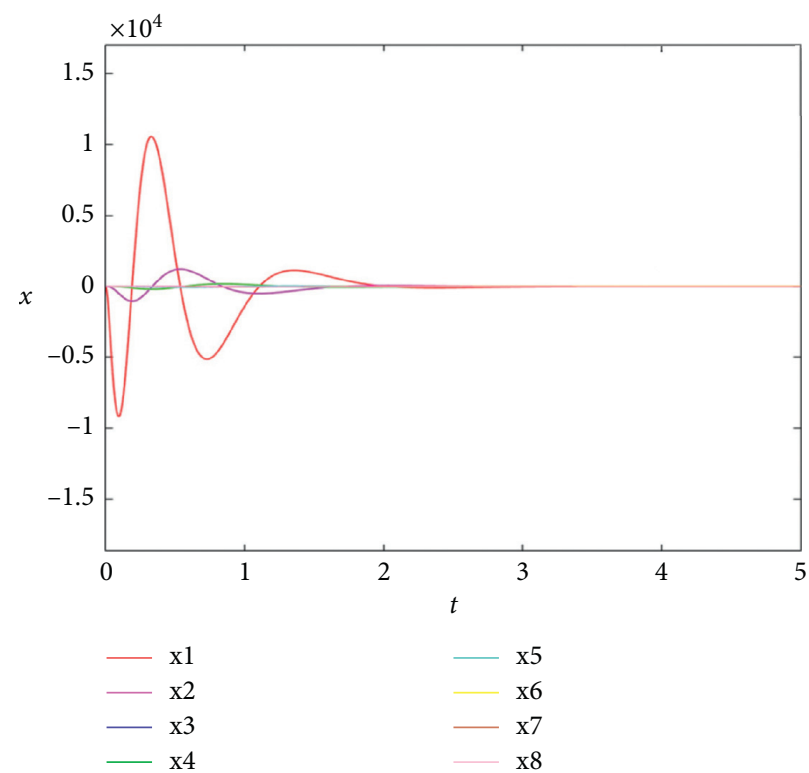

FIgURE 6: The different color curves in the figure represent the trend that the states of the 8 nodes in the new directed network change over time.

$$
C_{B}(v)=\sum_{s \neq v \neq t \in V} \frac{\sigma_{s t}(v)}{\sigma_{s t}}
$$

where $C_{B}(v)$ denotes the betweenness centrality of node $v, \sigma_{s t}(v)$ denotes the number of the shortest directed paths $(s \longrightarrow t)$ that passes through node $v$, and $\sigma_{s t}$ means the number of the shortest directed paths from node $s$ to node $t$.

Step 2. There is a nonlinear relationship between node load and node capacity described by

$$
\operatorname{Cap}(v)=C_{B}(v)+\beta\left(C_{B}(v)\right)^{\alpha}, \quad v=1,2, \ldots, n,
$$

where $\operatorname{Cap}(v)$ is the capacity of node $v, \alpha>0, \beta>0$. Since there is a positive correlation between node load and capacity, set $\alpha=\beta=1$. Thus, the node capacity is determined by

$$
\operatorname{Cap}(v)=2 C_{B}(v)
$$

Step 3. Use the index of node capacity to measure the node cost

$$
\operatorname{Cost}(v)=\operatorname{Cap}(v)=2 C_{B}(v),
$$

where $\operatorname{Cost}(v)$ denotes the cost of node $v$.

Step 4. Compare the capacities of two nodes of an edge, and take the larger one as the capacity of the edge (edge cost)

$$
\operatorname{Cost}\left(l_{i j}\right)=\max \left\{\operatorname{Cap}\left(v_{i}\right), \operatorname{Cap}\left(v_{j}\right)\right\},
$$

where $\operatorname{Cost}\left(l_{i j}\right)$ is the cost of edge $l_{i j}$.

Step 5. Calculate the network cost of each configuration according to Step 4

$$
\operatorname{Cost}(\mathrm{Net})=\sum \operatorname{Cost}\left(l_{i j}\right),
$$

where Cost(Net) denotes the cost of the whole network.

5.2. Data Processing. In Figure 4(a), the initial directed network $G(A, B)$ is given. Get the optimal edge-addition configuration by Algorithm 3, $\widetilde{E}_{1}^{*}=\left\{\left(x_{2}, x_{4}\right),\left(x_{5}, x_{6}\right),\left(x_{3}\right.\right.$, 
TABLE 1: The original data of betweenness centrality of each node in Figure 5.

\begin{tabular}{lcc}
\hline Node & Val & Label \\
\hline 1 & 0.47619051 & 1 \\
2 & 0.4761905 & 2 \\
3 & 0.3095238 & 3 \\
4 & 0.4761905 & 4 \\
5 & 0.3333333 & 5 \\
6 & 0.3333333 & 6 \\
7 & 0.1904762 & 7 \\
8 & 0.1904762 & 8 \\
\hline
\end{tabular}

We calculate the betweenness centrality value of eight nodes in Figure 5 by pajek software.

TABle 2: The data of node load, node capacity, edge cost, and network cost for the first configuration scheme.

\begin{tabular}{cccc}
\hline Edge & Node load & Node capacity & Edge cost \\
\hline$(1,2)$ & $(0.48,0.48)$ & $(0.96,0.96)$ & 0.96 \\
$(3,1)$ & $(0.31,0.48)$ & $(0.62,0.96)$ & 0.96 \\
$(2,4)$ & $(0.48,0.48)$ & $(0.96,0.96)$ & 0.96 \\
$(4,3)$ & $(0.48,0.31)$ & $(0.96,0.62)$ & 0.96 \\
$(4,5)$ & $(0.48,0.33)$ & $(0.96,0.66)$ & 0.96 \\
$(6,1)$ & $(0.33,0.48)$ & $(0.66,0.96)$ & 0.96 \\
$(5,6)$ & $(0.33,0.33)$ & $(0.66,0.66)$ & 0.66 \\
$(3,7)$ & $(0.31,0.19)$ & $(0.62,0.38)$ & 0.62 \\
$(7,8)$ & $(0.19,0.19)$ & $(0.38,0.38)$ & 0.38 \\
$(8,7)$ & $(0.19,0.19)$ & $(0.38,0.38)$ & 0.38 \\
$(8,5)$ & $(0.19,0.33)$ & $(0.38,0.66)$ & 0.66 \\
& Network cost & & 8.46 \\
\hline
\end{tabular}

$\left.\left.x_{7}\right)\right\}$ and $\widetilde{E}_{2}^{*}=\left\{\left(x_{2}, x_{4}\right),\left(x_{5}, x_{7}\right),\left(x_{3}, x_{6}\right)\right\}$ and $\widetilde{E}_{3}^{*}=\left\{\left(x_{2}\right.\right.$, $\left.\left.x_{4}\right),\left(x_{5}, x_{6}\right),\left(x_{6}, x_{7}\right)\right\}$ and $\widetilde{E}_{4}^{*}=\left\{\left(x_{2}, x_{4}\right),\left(x_{6}, x_{7}\right),\left(x_{3}, x_{6}\right)\right\}$.

The new directed network resulting from the first configuration scheme is shown in Figure 5. Figure 6 shows the curve of the state of each node over time.

We import this new directed network $G(A+\widetilde{A}, B)$ into pajek software to calculate the betweenness centrality of each node. The original data of betweenness centrality of each node are shown in Table 1. In Table 2, we collate the data of node load, node capacity, edge cost, and network cost according to each step described in Section 5.1. Then, we get the network cost of the first configuration scheme.

The new directed network resulting from the second configuration scheme is shown in Figure 7. The original data of betweenness centrality of each node are shown in Table 3. Similarly, we can obtain the data of node load, node capacity, edge cost, and network cost, as shown in Table 4.

The new directed network resulting from the third configuration scheme is shown in Figure 8. The original data of betweenness centrality of each node are shown in Table 5 . Similarly, we can obtain the data of node load, node capacity, edge cost, and network cost, as shown in Table 6.

The new directed network resulting from the fourth configuration scheme is shown in Figure 9. The original data of betweenness centrality of each node are shown in Table 7. Furthermore, we can obtain the data of node load, node capacity, edge cost, and network cost, as shown in Table 8.

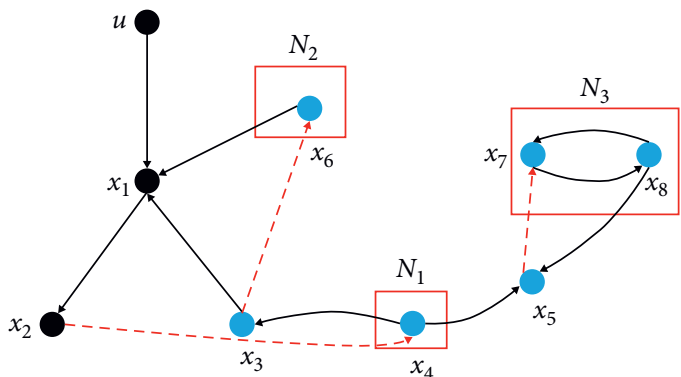

FIgURE 7: The new directed network resulting from the second configuration scheme $\widetilde{E}_{2}^{*}=\left\{\left(x_{2}, x_{4}\right),\left(x_{5}, x_{7}\right),\left(x_{3}, x_{6}\right)\right\}$.

TABLE 3: The original data of betweenness centrality of each node in Figure 7.

\begin{tabular}{lcc}
\hline Node & Val & Label \\
\hline 1 & 0.2857143 & 1 \\
2 & 0.3571429 & 2 \\
3 & 0.1428571 & 3 \\
4 & 0.4285714 & 4 \\
5 & 0.2380952 & 5 \\
6 & 0.0000000 & 6 \\
7 & 0.1428571 & 7 \\
8 & 0.0238095 & 8 \\
\hline We calculate the betweenness centrality value of eight nodes in Figure 7 by \\
pajek software
\end{tabular}

TABle 4: The data of node load, node capacity, edge cost, and network cost for the second configuration scheme.

\begin{tabular}{cccc}
\hline Edge & Node load & Node capacity & Edge cost \\
\hline$(1,2)$ & $(0.29,0.36)$ & $(0.58,0.72)$ & 0.72 \\
$(3,1)$ & $(0.14,0.29)$ & $(0.28,0.58)$ & 0.58 \\
$(2,4)$ & $(0.36,0.43)$ & $(0.72,0.86)$ & 0.86 \\
$(4,3)$ & $(0.43,0.14)$ & $(0.86,0.28)$ & 0.86 \\
$(4,5)$ & $(0.43,0.24)$ & $(0.86,0.48)$ & 0.86 \\
$(6,1)$ & $(0.00,0.29)$ & $(0.00,0.58)$ & 0.58 \\
$(5,7)$ & $(0.24,0.14)$ & $(0.48,0.24)$ & 0.48 \\
$(3,6)$ & $(0.14,0.00)$ & $(0.28,0.00)$ & 0.28 \\
$(7,8)$ & $(0.14,0.02)$ & $(0.28,0.04)$ & 0.28 \\
$(8,7)$ & $(0.02,0.14)$ & $(0.04,0.28)$ & 0.28 \\
$(8,5)$ & $(0.02,0.24)$ & $(0.04,0.48)$ & 0.48 \\
& Network cost & & 6.26 \\
\hline
\end{tabular}

Comparing the network costs of the above four configuration schemes, we choose the fourth scheme as the optimal edge-addition configuration so as to get the solution of Problem 3.

5.3. Illustrative Example. In [23], a directed network as shown in Figure 10 is considered. The authors proposed 14 edge-addition configurations, i.e., $\widetilde{E}^{*}=\left\{\left(x_{2}, x_{10}\right),\left(x_{9}, x_{5}\right)\right.$, $\left.\left(x_{i}, x_{j}\right)\right\}, i \in\{1, \ldots, 6,10\}, j \in\{7,8\}$. However, they did not tell us which one is the optimal edge-addition configuration with the lowest cost. Using the results of our work, the cost of each optimization scheme can be calculated, and finally a scheme $\widetilde{E}_{8}^{*}=\left\{\left(x_{2}, x_{10}\right),\left(x_{9}, x_{5}\right),\left(x_{4}, x_{8}\right)\right\}$ with the lowest 




Figure 8: The new directed network resulting from the third configuration scheme $\widetilde{E}_{3}^{*}=\left\{\left(x_{2}, x_{4}\right),\left(x_{5}, x_{6}\right),\left(x_{6}, x_{7}\right)\right\}$.

TABLE 5: The original data of betweenness centrality of each node in Figure 8.

\begin{tabular}{lcc}
\hline Node & Val & Label \\
\hline 1 & 0.4523810 & 1 \\
2 & 0.4523810 & 2 \\
3 & 0.0714286 & 3 \\
4 & 0.4523810 & 4 \\
5 & 0.5238095 & 5 \\
6 & 0.5238095 & 6 \\
7 & 0.1666667 & 7 \\
8 & 0.1666667 & 8 \\
\hline
\end{tabular}

We calculate the betweenness centrality value of eight nodes in Figure 8 by pajek software.

TABle 6: The data of node load, node capacity, edge cost, and network cost for the third configuration scheme.

\begin{tabular}{cccc}
\hline Edge & Node load & Node capacity & Edge cost \\
\hline$(1,2)$ & $(0.45,0.45)$ & $(0.90,0.90)$ & 0.90 \\
$(3,1)$ & $(0.07,0.45)$ & $(0.14,0.90)$ & 0.90 \\
$(2,4)$ & $(0.45,0.45)$ & $(0.90,0.90)$ & 0.90 \\
$(4,3)$ & $(0.45,0.07)$ & $(0.90,0.14)$ & 0.90 \\
$(4,5)$ & $(0.45,0.52)$ & $(0.90,1.04)$ & 1.04 \\
$(6,1)$ & $(0.52,0.45)$ & $(1.04,0.90)$ & 1.04 \\
$(5,6)$ & $(0.52,0.52)$ & $(1.04,1.04)$ & 1.04 \\
$(6,7)$ & $(0.52,0.17)$ & $(1.04,0.34)$ & 1.04 \\
$(7,8)$ & $(0.17,0.17)$ & $(0.34,0.34)$ & 0.34 \\
$(8,7)$ & $(0.17,0.17)$ & $(0.34,0.34)$ & 0.34 \\
$(8,5)$ & $(0.17,0.52)$ & $(0.34,1.04)$ & 1.04 \\
& Network cost & & 9.48 \\
\hline
\end{tabular}

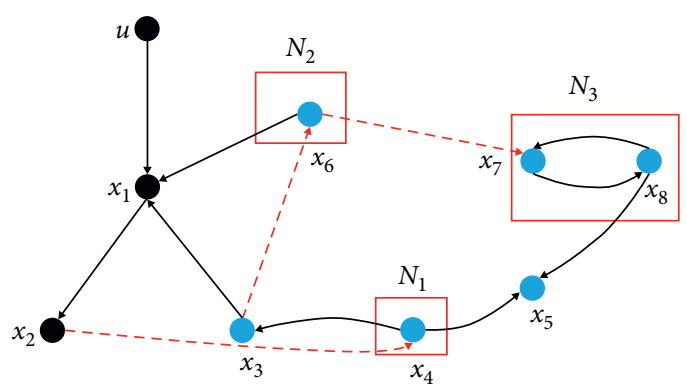

FIgURE 9: The new directed network resulting from the fourth configuration scheme $\widetilde{E}_{4}^{*}=\left\{\left(x_{2}, x_{4}\right),\left(x_{6}, x_{7}\right),\left(x_{3}, x_{6}\right)\right\}$.
TABLE 7: The original data of betweenness centrality of each node in Figure 9.

\begin{tabular}{lcc}
\hline Node & Val & Label \\
\hline 1 & 0.1547619 & 1 \\
2 & 0.2261905 & 2 \\
3 & 0.2857143 & 3 \\
4 & 0.2976190 & 4 \\
5 & 0.0000000 & 5 \\
6 & 0.2023810 & 6 \\
7 & 0.1547619 & 7 \\
8 & 0.0595238 & 8 \\
\hline
\end{tabular}

We calculate the betweenness centrality value of eight nodes in Figure 9 by pajek software.

TABle 8: The data of node load, node capacity, edge cost, and network cost for the fourth configuration scheme.

\begin{tabular}{lccc}
\hline Edge & Node load & Node capacity & Edge cost \\
\hline$(1,2)$ & $(0.15,0.23)$ & $(0.30,0.46)$ & 0.46 \\
$(3,1)$ & $(0.29,0.15)$ & $(0.58,0.30)$ & 0.58 \\
$(2,4)$ & $(0.23,0.30)$ & $(0.46,0.60)$ & 0.60 \\
$(4,3)$ & $(0.30,0.29)$ & $(0.60,0.58)$ & 0.60 \\
$(4,5)$ & $(0.30,0.00)$ & $(0.60,0.00)$ & 0.60 \\
$(6,1)$ & $(0.20,0.15)$ & $(0.40,0.30)$ & 0.40 \\
$(3,6)$ & $(0.29,0.20)$ & $(0.58,0.40)$ & 0.58 \\
$(6,7)$ & $(0.20,0.15)$ & $(0.40,0.30)$ & 0.40 \\
$(7,8)$ & $(0.15,0.06)$ & $(0.30,0.12)$ & 0.30 \\
$(8,7)$ & $(0.06,0.15)$ & $(0.12,0.30)$ & 0.30 \\
$(8,5)$ & $(0.06,0.00)$ & $(0.12,0.00)$ & 0.12 \\
\multicolumn{5}{c}{ Network cost } & & 4.94 \\
\hline
\end{tabular}



Figure 10: A directed network.

cost can be selected to ensure the structural controllability of the network.

\section{Conclusions}

In this paper, we have solved the problem of how to optimize the network topology to ensure structural controllability. Given a structurally uncontrollable directed network, Algorithm 3 presents all possible edge-addition configurations. After determining the optimal edge-addition configuration, a network cost index is given to choose the lowest cost configuration.

In future, we can combine these two strategies of adding edges and adding external input signals to ensure the network controllability and choose the scheme with the highest benefit by comparing the costs of several strategies. In 
addition, we can extend a single directed network to the topology design of a multiplex network $[29,41]$ so as to ensure the structural controllability of the multiplex network.

\section{Data Availability}

No data were used to support this study.

\section{Conflicts of Interest}

The authors declare that they have no conflicts of interest.

\section{Acknowledgments}

This work was supported in part by the National Natural Science Foundation of China under grant no. 61973064, the Natural Science Foundation of Hebei Province of China under grant no. F2019501126, the Natural Science Foundation of Liaoning Province of China under grant no. 2020KF-11-03, and the Fundamental Research Funds for the Central Universities under grant no. N182304013.

\section{References}

[1] R. E. Kalman, "Mathematical description of linear dynamical systems," Journal of the Society for Industrial and Applied Mathematics Series A Control, vol. 1, no. 2, pp. 152-192, 1963.

[2] Y.-Y. Liu, J.-J. Slotine, and A.-L. Barabási, "Controllability of complex networks," Nature, vol. 473, no. 7346, pp. 167-173, 2011.

[3] Y.-Y. Liu and A.-L. Barabási, "Control principles of complex systems," Reviews of Modern Physics, vol. 88, Article ID 35006, 2016.

[4] N. Cai and Y.-S. Zhong, "Formation controllability of highorder linear time-invariant swarm systems," IET Control Theory \& Applications, vol. 4, no. 4, pp. 646-654, 2010.

[5] C. Commault, "Structural controllability of networks with dynamical structured nodes," IEEE Transactions on Automatic Control, vol. 65, no. 6, pp. 2736-2742, 2020.

[6] Y. Y. Yang, "Research progress in enhancing the controllability of complex networks," Discrete Dynamics in Nature and Society, vol. 2020, Article ID 5759264, 8 pages, 2020.

[7] B. Liu, H. Su, L. Wu, and X. Shen, "Observability of leaderbased discrete-time multi-agent systems over signed networks," IEEE Transactions on Network Science and Engineering, vol. 1, 2020.

[8] W. Wang, F. Chen, L. Xiang, and G. Chen, "A distributed algorithm for tracking general functions of multiple signals not-necessarily having steady states," IEEE Transactions on Circuits and Systems II: Express Briefs, vol. 1, 2020.

[9] I. Rajapakse, M. Groudine, and M. Mesbahi, "Dynamics and control of state-dependent networks for probing genomic organization," Proceedings of the National Academy of Sciences, vol. 108, no. 42, pp. 17257-17262, 2011.

[10] B. Liu, X. Shen, L. Wu, and H. Su, "Observability of leaderbased discrete-time multi-agent systems with switching topology," IET Control Theory \& Applications, vol. 14, no. 16, pp. 2462-2471, 2020.

[11] F. Chen, X. Z. Chen, L. Y. Xiang, and W. Ren, "Distributed economic dispatch via a predictive scheme: heterogeneous delays and privacy preservation," Automatica, vol. 123, Article ID 109356, 2020.
[12] L. Mo and S. Guo, "Consensus of linear multi-agent systems with persistent disturbances via distributed output feedback," Journal of Systems Science and Complexity, vol. 32, no. 3, pp. 835-845, 2019.

[13] S. Liu, Z. J. Ji, and H. Z. Ma, "Jordan form-based algebraic conditions for controllability of multiagent systems under directed graphs," Complexity, vol. 2020, Article ID 7685460, 18 pages, 2020.

[14] Z. Ji, H. Lin, S. Cao, Q. Qi, and H. Ma, "The complexity in complete graphic characterizations of multiagent controllability," IEEE Transactions on Cybernetics, vol. 51, no. 1, pp. 64-76, 2021.

[15] F. Chen and W. Ren, "Sign projected gradient flow: a continuous-time approach to convex optimization with linear equality constraints," Automatica, vol. 120, Article ID 109156, 2020.

[16] L. Xiang, F. Chen, W. Ren, and G. Chen, "Advances in network controllability," IEEE Circuits and Systems Magazine, vol. 19, no. 2, pp. 8-32, 2019.

[17] L. Xiang, P. Wang, F. Chen, and G. Chen, "Controllability of directed networked MIMO systems with heterogeneous dynamics," IEEE Transactions on Control of Network Systems, vol. 7, no. 2, pp. 807-817, 2020.

[18] Z. Z. Yuan, C. Zhao, Z. R. Di, W.-X. Wang, and Y.-C. Lai, "Exact controllability of complex networks," Nature Communications, vol. 4, p. 2447, 2013.

[19] T. Nepusz and T. Vicsek, "Controlling edge dynamics in complex networks," Nature Physics, vol. 8, no. 7, pp. 568-573, 2012.

[20] S.-P. Pang, W.-X. Wang, F. Hao, and Y.-C. Lai, "Universal framework for edge controllability of complex networks," Scientific Reports, vol. 7, p. 4224, 2017.

[21] L. Xiang and G. Chen, "Minimal edge controllability of directed networks," Advances in Complex Systems, vol. 22, Article ID 1950017, 2019.

[22] W.-X. Wang, X. Ni, Y.-C. Lai, and C. Grebogi, "Optimizing controllability of complex networks by minimum structural perturbations," Physical Review E, vol. 85, no. 2, Article ID 26115, 2012.

[23] X. Chen, S. Pequito, G. J. Pappas, and V. M. Preciado, "Minimal edge addition for network controllability," IEEE Transactions on Control of Network Systems, vol. 6, no. 1, pp. 312-323, 2019.

[24] Y. Zhang and T. Zhou, "Minimal structural perturbations for controllability of a networked system: complexities and approximations," International Journal of Robust and Nonlinear Control, vol. 29, no. 7291, pp. 4191-4208, 2019.

[25] G. Yan, J. Ren, Y.-C. Lai, C.-H. Lai, and B. Li, "Controlling complex networks: how much energy is needed?" Physical Review Letters, vol. 108, Article ID 218703, 2002.

[26] F. Chen and J. Chen, "Minimum-energy distributed consensus control of multiagent systems: a network approximation approach," IEEE Transactions on Automatic Control, vol. 65, no. 3, pp. 1144-1159, 2020.

[27] Z. H. Zhang, Y. F. Yin, and X. Zhang, "Optimization of robustness of interdependent network controllability by redundant design," PLoS One, vol. 13, no. 2, Article ID e0192874, 2018.

[28] Y. Lou, L. Wang, and G. Chen, "Enhancing controllability robustness of q-snapback networks through redirecting edges," Research, vol. 11, Article ID 7857534, 2019.

[29] M. Posfai, J. Gao, and S. P. Cornelius, "Controllability of multiplex, multitime-scale networks," Physical Review E, vol. 94, no. 3, Article ID 32316, 2016. 
[30] L. Z. Wang, Y. Z. Chen, W.-X. Wang, and Y.-C. Lai, "Physical controllability of complex networks," Scientific Reports, vol. 7, Article ID 40198, 2017.

[31] C.-T. Lin, "Structural controllability," IEEE Transactions on Automatic Control, vol. 19, no. 3, pp. 201-208, 1974.

[32] R. Shields and J. Pearson, "Structural controllability of multiinput linear systems," IEEE Transactions on Automatic Control, vol. 21, no. 2, pp. 203-212, 1976.

[33] K. Glover and L. Silverman, "Characterization of structural controllability," IEEE Transactions on Automatic Control, vol. 21, no. 4, pp. 534-537, 1976.

[34] J.-M. Dion, C. Commault, and J. van der Woude, "Generic properties and control of linear structured systems: a survey," Automatica, vol. 39, no. 7, pp. 1125-1144, 2003.

[35] C. Sueur and G. Dauphin-Tanguy, "Bond-graph approach for structural analysis of MIMO linear systems," Journal of the Franklin Institute, vol. 328, no. 1, pp. 55-70, 1991.

[36] K. Murota and S. Poljak, "Note on a graph-theoretic criterion for structural output controllability," IEEE Transactions on Automatic Control, vol. 35, no. 8, pp. 939-942, 1990.

[37] C. Commault, J. M. Dion, and J. W. V. D. Woude, "Characterization of generic properties of linear structured systems for efficient computations," Kybernetika, vol. 38, no. 5, pp. 503-520, 2002.

[38] D.-H. Kim and A. E. Motter, "Resource allocation pattern in infrastructure networks," Journal of Physics A: Mathematical and Theoretical, vol. 41, no. 22, Article ID 224019, 2008.

[39] B.-L. Dou, X.-G. Wang, and S.-Y. Zhang, "Robustness of networks against cascading failures," Physica A: Statistical Mechanics and its Applications, vol. 389, no. 11, pp. 23102317, 2010.

[40] L. J. Liu, Y. F. Yin, Z. H. Zhang, and Y. K. Malaiya, "Redundant design in interdependent networks," PLoS One, vol. 11, no. 10, Article ID e0164777, 2016.

[41] Z. Wang, C. Xia, Z. Chen, and G. Chen, "Epidemic propagation with positive and negative preventive information in multiplex networks," IEEE Transactions on Cybernetics, vol. 1, 2020. 